\title{
Evaluation of Thoracic Kyphosis Angle and Respiratory Functions in Patients with Multiple Sclerosis
}

\author{
${ }^{1 \infty}$ Musa Muhtaroglu, ${ }^{2 \infty}$ Sevda Lafcı Fahrioglu*, ${ }^{3}$ Ferda Selcuk, ${ }^{2}$ Sezgin İlgi \\ ${ }^{1}$ Physiotherapy Clinic, Dr. Burhan Nalbantoğlu State Hospital, Nicosia, Cyprus. ${ }^{2}$ Faculty of Medicine, \\ Departments of Anatomy, Near East University, Nicosia, Cyprus. ${ }^{3}$ Departments of Neurology, Dr. Burhan \\ Nalbantoğlu State Hospital, Nicosia, Cyprus.
}

Submitted 02 February 2021; Accepted in final form 25 March 2021.

\begin{abstract}
Background. Multiple Sclerosis (MS) is a chronic autoimmune demyelinating disease. Muscle weakness, tremors, balance disorders, and gait instability are observed in MS patients. Also, the respiratory muscles' weakness can lead to decreased respiratory capacity, resulting in pneumonia and acute respiratory failure. Objectives. This study evaluates thoracic kyphosis angle (TKA) and respiratory functions in MS patients and healthy groups. Methods. The study consists of 40 patients with MS (mean age of $39 \pm 7.89$ years, mean height of $167.5 \pm 10.19 \mathrm{~cm}$, mean weight of 73.12 $\pm 14.40 \mathrm{~kg}$ ) and 41 healthy subjects (mean age of $37.12 \pm 8.89$ years, mean height of $167.9 \pm 10.76 \mathrm{~cm}$, mean weight of $72.7 \pm 16.91 \mathrm{~kg}$ ). Respiratory function tests and TKA measurements were performed. Results. There was a statistically significant difference between TKA measurements in MS patients and healthy subjects $(\mathrm{p}<0.05)$. The respiratory function test values in the healthy subjects were also significantly higher than in the MS patients $(\mathrm{p}<0.05)$. Conclusion. Changes in respiratory function due to muscle weakness and altered posture and TKA in MS patients should be considered in treatment.
\end{abstract}

KEYWORDS: Multiple Sclerosis, Pulmonary Function Test, Thoracic Kyphosis Angle, Anthropometry.

\section{INTRODUCTION}

Multiple Sclerosis (MS) is a chronic, inflammatory, demyelinating disease of the central nervous system (1). It affects approximately 2.5 million people in the world (2). MS usually progresses in episodes and subsequently leads to a progressive degenerative process. As a result, it severely limits the quality of life of patients and causes progressive disability. Due to axonal damage, muscle spasms, tremors, balance disturbances, gait instability, and fatigue are the primary motor symptoms observed by MS patients $(3,4)$. Motor impairments lead to limited mobility and low physical activity, resulting in further disability (3). As much as the clinical condition of the disease allows, being physically active is very important to reduce the occurrence of comorbidities so that patients with multiple sclerosis can minimize the loss of their functional abilities. The degree of their overall neurological impairment is assessed using the Expanded Disability Status Scale (EDSS) (1). The EDSS, the most commonly used disability scale for MS patients, provides an effective and reliable assessment at any disease stage (2). Fatigue, impaired body control, especially with the hamstring and quadriceps muscles' weakness, cause patients to have gait disturbances, a decrease in walking speed and stride length, and a decrease

$\infty$. These authors contributed equally to this study.

*. Corresponding Author:

Sevda Lafeı Fahrioglu, Associate Professor

E-mail: sevdalafci@gmail.com 
in postural stability. Muscle weakness also causes abnormal curvatures of the spine and postural changes due to decreased upper extremity function (5). Kyphosis, which is called the thoracic vertebrae's anterior curvature, is caused by the structures of their body, the intervertebral disk, and the paravertebral muscle's strength.

MS Patients with gait disorder have to lean forward to see their steps, and this can cause kyphosis anomaly (6). It leads to an increased risk of falls and fractures, physical dysfunction, and poor life quality. There are few studies in the literature examining the relationship between hyperkyphosis and respiratory function (6). A 2020 study reported increased thoracic kyphosis angle (TKA) might lead to pulmonary function limitation (7). Measurement of TKA using a digital inclinometer is a non-invasive technique that is also valid and reliable. It can be used for regular screening (7). The course of progressive disabilities varies according to the nature and duration of the disease.

In addition to musculoskeletal and neurological problems, dysphagia, respiratory muscle weakness, and resulting respiratory failure are important causes of mortality in MS patients (8). Respiratory issues are pretty standard, especially in the advanced stages of MS. Aspiration and pneumonia increase morbidity in more severe stages in half of MS patients $(1,9)$. Respiratory workload increases as a result of impaired lung mechanics. Shortness of breath, which occurs even in patients with a regular respiratory function test, can be explained by decreased respiratory muscle strength in patients. It has been reported that functional exercise capacity is lower in MS patients (8). Monitoring of respiratory function tests in MS patients is essential to determine the progression of the disease. It has been reported that disturbances in respiratory dynamics become apparent, especially during physical exertion in further stages MS patients. In these cases, the impaired respiratory function has severe implications for morbidity and mortality. It has been reported that the cause of death is pneumonia and influenza in $20 \%$ of MS patients under 50 years of age $(8,9)$. Moreover, the number of studies on the impact of early respiratory function in the literature is limited, and prevalence data are insufficient (9, 10). Although there are studies in the literature that evaluate MS patients' pulmonary functions, our study is a new study that assesses MS patients' respiratory functions along with the thoracic kyphosis angle measured by a digital inclinometer.

\section{MATERIALS AND METHODS}

Research Method and Ethical

Consideration. This study was conducted between August 2018 and December 2019 in the Neurology Clinic of Dr. Burhan Nalbantoglu State Hospital. In the study, pulmonary function tests were performed, and TKA was measured in the patients and the control group. The study's ethics committee approval was obtained from the Scientific Research and Publication Ethics Board of Dr. Burhan Nalbantoğlu State Hospital (dated 20.06.2018 and decision number 2018 / 25-12).

Participants. Considering that the samples could be excluded from the study for various reasons, 50 cases were reached for the task. Ten MS patients were not included in the study. Of the patients who came, six could not fulfill the requirements (they were smokers, had neurological, orthopedic, or chronic diseases such as heart failure and respiratory problems, were pregnant, or did not agree to participate), and 4 had an EDSS above 6. Forty patients diagnosed by the neurologist as having the relapsingremitting type of MS according to the McDonald diagnostic criteria were included. Patients were non-smokers with oxygen saturation scores above 95 and with EDSS scores of 0-5, aged between 18 and 55. Forty-one healthy non-smoking volunteers with similar physical characteristics were also included.

Tolls and Measurements. After the neurologist's neurological examination, the respiratory physiotherapist and the anatomist performed the anthropometric and clinometric evaluations of the spine and respiratory functions. Pulmonary function tests including forced vital capacity (FVC), expired air volume in the first second of forced expiration (FEV1), FEV1 / FVC, peak expiratory flow (PEF), and forced expiratory flow between $25 \%$ and $75 \%$ of vital capacity, FEF25-75 were evaluated using percentages. Participants' TKA measurements were obtained using two digital inclinometer devices (Baseline ${ }^{\circledR}$ Digital Inclinometer). Participants were asked to keep both lower extremities in the same position, arms in a free and natural position, and look forward comfortably. The spinous process of $\mathrm{C} 7$ was determined by palpation while the participants' 
heads were placed in the flexion position, and T1 was identified below the spinous process. The spinous process of $\mathrm{T} 12$ was marked by palpation upward from the fifth lumbar vertebra. TKA was measured by attaching one end of the dual digital inclinometer to T1 and T12. The value seen on the screen of the digital inclinometer was recorded. The average of three measurements taken by the same investigator was defined as TKA. The anthropometric parameters shown in Table 2 were measured using digital equipment and a flexible ruler. All subjects were informed of all details of the study procedures and completed a written informed consent form before the evaluations.

Study Protocols. MS patients were divided into two groups: Group I corresponding to mild MSS (EDSS or $=2.5$ ), Group II corresponding to moderate MSS (EDSS 2.5-5). The data of the two groups were compared with each other as well as with the control group.

Statistical Evaluation. Statistical Package for Social Sciences (SPSS) software version 25 and independent t-test were used for data analysis at the significance level of $\mathrm{p}<0.05$.

\section{RESULTS}

The study consists of 40 patients with MS (mean age of $39 \pm 7.89$ years, mean height of $167.5 \pm 10.19 \mathrm{~cm}$, mean weight of $73.12 \pm 14.40$ $\mathrm{kg}$ ), and 41 healthy subjects (mean age of $37.12 \pm 8.89$ years, mean height of $167.9 \pm 10.76$ $\mathrm{cm}$, mean weight of $72.7 \pm 16.91 \mathrm{~kg}$ ). In our study, conducted on 28 women (70\%) with MS and 29 healthy women $(70.73 \%), 27.5 \%$ were under the age of $30,22.55 \%$ were between the ages of 30 $39,35 \%$ were between the ages of $40-49,15 \%$ were in the age group of $>50$ years (Table 1-2). It was evaluated that $47.5 \%$ of MS patients included in group I, and $52.5 \%$ of them were evaluated in group II (Figure 1). The duration of the disease in MS patients was found as $8.75 \pm 6.23$ (min-max:125 ) years, $35 \%$ of the MS patients included in the study had a 10 -year disease period and above, while $65 \%$ had a disease duration of 10 years or below (Figure 2).

There was a statistically significant difference in the anthropometric measurements only in chest circumference of MS patients and the healthy group (98.23 cm vs. $94.17 \mathrm{~cm}$, respectively, $\mathrm{p}<0.046$ ). The rest of the anthropometric measurements of MS patients and the healthy group were found to be statistically similar (Table 2).

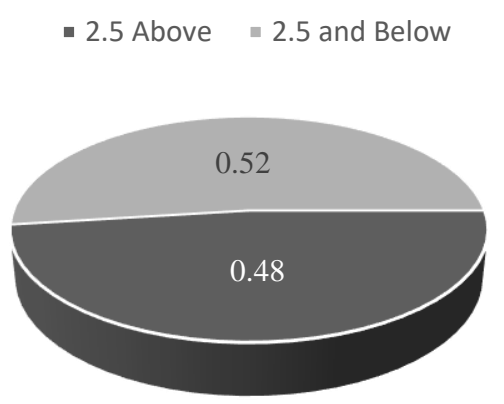

Figure 1. Distribution of MS patients by EDSS scores Group I: EDSS < or $=2.5$

Group II: EDSS above 2.5

- 10 Years Above - 10 Years and Below

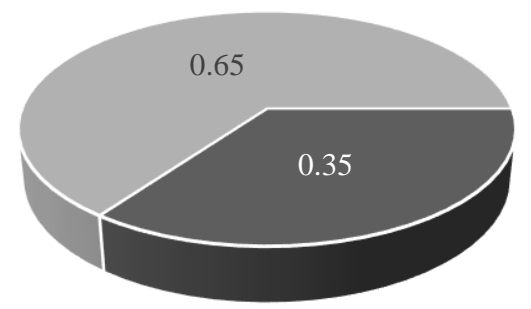

Figure 2. Distribution of MS patients by disease duration

The respiratory function test results were higher in a healthy group with statistically significant $(p<0.05)$. The TKA measurements of MS patients were significantly higher than those of the healthy volunteers (Table 2).

The TKA and pulmonary function test results in Group I MS patients and healthy subjects were compared. It was found that TKA values of Group I patients were statistically significantly higher than healthy individuals $\left(41.56^{\circ}\right.$ vs. $35.70^{\circ}$, respectively, $p=0.003)$. In the comparison of respiratory functions of Group I MS patients and healthy volunteers, the FEV1/FVC $(\%)$ of the healthy volunteers were significantly higher than Group I MS (84.63\% vs. $80.28 \%$ respectively, $\mathrm{p}=$ 0,021 ) (Table 3). In the comparison of Group II MS and healthy individuals, it was detected that there was a statistically significant difference between the values of FVC (L) (\%) FEV1 (L) (\%), FEV1/FVC (\%) (measured), PEF (L/s) (\%), FEF $25-75(\mathrm{~L} / \mathrm{s})(\%) \quad(\mathrm{p}<0.05)$. The respiratory functions mentioned above of healthy volunteers were high, but the TKA measurement value was low (Table 3). Also, when Group I and Group II MS patients' pulmonary function tests were compared, it was found that the values of Group I 
were statistically significantly higher than Group II, except for FEV1 / FVC. The TKA measurements were significantly higher in Group II MS than in Group I MS (Table 3).

Table 1. Demographic data of MS patients and healthy volunteers

\begin{tabular}{|c|c|c|c|c|}
\hline & \multicolumn{2}{|c|}{ MS $(n=40)$} & \multicolumn{2}{|c|}{ Healthy $(n=41)$} \\
\hline & $\mathbf{n}$ & $\%$ & $\mathbf{n}$ & $\%$ \\
\hline \multicolumn{5}{|l|}{ Gender } \\
\hline Female & 28 & 70.00 & 29 & 70.73 \\
\hline Male & 12 & 30.00 & 12 & 29.27 \\
\hline \multicolumn{5}{|l|}{ Age (year) } \\
\hline$<30$ & 11 & 27.50 & 10 & 24.39 \\
\hline $30-39$ & 9 & 22.50 & 15 & 36.59 \\
\hline $40-49$ & 14 & 35.00 & 9 & 21.95 \\
\hline $50>$ & 6 & 15.00 & 7 & 17.07 \\
\hline
\end{tabular}

Table 2. Comparison of anthropometric measurements of MS patients and healthy volunteers

\begin{tabular}{|c|c|c|c|c|c|}
\hline 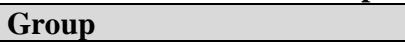 & No. & Mean \pm SD & $\min$ & $\max$ & p \\
\hline Height (cm) & & & & & 1.000 \\
\hline MS & 40 & $167.55 \pm 41.00$ & 145.00 & 190.00 & \\
\hline Healthy & 41 & $167.90 \pm 41.00$ & 150.00 & 189.00 & \\
\hline Weight (kg) & & & & & 0.913 \\
\hline MS & 40 & $73.13 \pm 41.29$ & 50.00 & 100.00 & \\
\hline Healthy & 41 & $72.71 \pm 40.72$ & 46.00 & 120.00 & \\
\hline BMI $\left(\mathrm{kg} / \mathrm{m}^{2}\right)$ & & & & & 0.603 \\
\hline MS & 40 & $26.04 \pm 42.38$ & 18.37 & 40.57 & \\
\hline Healthy & 41 & $25.67 \pm 39.66$ & 19.10 & 37.87 & \\
\hline Neck circumference (cm) & & & & & 0.222 \\
\hline MS & 40 & $35.25 \pm 44.23$ & 29.00 & 43.00 & \\
\hline Healthy & 41 & $34.02 \pm 37.85$ & 27.00 & 44.00 & \\
\hline Shoulder circumference $(\mathrm{cm})$ & & & & & 0.321 \\
\hline MS & 40 & $104.34 \pm 43.63$ & 87.00 & 121.00 & \\
\hline Healthy & 41 & $103.29 \pm 38.44$ & 86.00 & 132.00 & \\
\hline Chest circumference $(\mathrm{cm})$ & & & & & $0.046^{*}$ \\
\hline MS & 40 & $98.23 \pm 46.29$ & 80.00 & 116.00 & \\
\hline Healthy & 41 & $94.17 \pm 35.84$ & 70.00 & 120.00 & \\
\hline A fathom length $(\mathrm{cm})$ & & & & & 0.795 \\
\hline MS & 40 & $169.14 \pm 41.69$ & 148.00 & 190.00 & \\
\hline Healthy & 41 & $168.73 \pm 40.33$ & 147.00 & 190.00 & \\
\hline
\end{tabular}

Table 3. Respiratory functions and TKA relation of healthy group and patients with MS

\begin{tabular}{|c|c|c|c|c|c|}
\hline Group & n & Mean \pm SD & Min & Max & $\mathbf{p}$ \\
\hline FVC (L) (\%) & & & & & $0.008 *$ \\
\hline MS & 40 & $90.15 \pm 33.96$ & 49.00 & 116.00 & \\
\hline Healthy & 41 & $97.98 \pm 47.87$ & 69.00 & 133.00 & \\
\hline $\operatorname{FEV1}(\mathrm{L})(\%)$ & & & & & $0.001 *$ \\
\hline MS & 40 & $87.13 \pm 32.23$ & 55.00 & 109.00 & \\
\hline Healthy & 41 & $96.41 \pm 49.56$ & 75.00 & 137.00 & \\
\hline FEV1/FVC (\%) (measured) & & & & & $0.001 *$ \\
\hline MS & 40 & $79.50 \pm 31.89$ & 69.00 & 90.00 & \\
\hline Healthy & 41 & $84.63 \pm 49.89$ & 72.00 & 99.00 & \\
\hline PEF (L/s) (\%) & & & & & $0.022 *$ \\
\hline MS & 40 & $78.50 \pm 34.95$ & 29.00 & 115.00 & \\
\hline Healthy & 41 & $88.02 \pm 46.90$ & 65.00 & 121.00 & \\
\hline FEF25-75 (L/s) (\%) & & & & & $0.001 *$ \\
\hline MS & 40 & $74.40 \pm 32.30$ & 33.00 & 117.00 & \\
\hline Healthy & 41 & $89.05 \pm 49.49$ & 38.00 & 135.00 & \\
\hline TKA & & & & & $0.000 *$ \\
\hline MS & 40 & $46.13 \pm 55.64$ & 28.70 & 59.00 & \\
\hline Healthy & 41 & $35.70 \pm 26.72$ & 22.30 & 49.20 & \\
\hline
\end{tabular}

* $\mathrm{p}<0.05$. Mann-Whitney U test was used. TKA: thoracic kyphosis angle 
Table 4. Comparison of respiratory functions and TKA angle of the Group I MS patients and healthy volunteers

\begin{tabular}{|c|c|c|c|c|c|}
\hline & $\mathbf{n}$ & $\%$ & Min & Max & $\mathbf{p}$ \\
\hline FVC (L) (\%) & & & & & 0.453 \\
\hline Group I & 21 & 96.00 & 82.00 & 116.00 & \\
\hline Healthy & 41 & 97.98 & 69.00 & 133.00 & \\
\hline FEV1 (L) (\%) & & & & & 0.369 \\
\hline Group I & 21 & 93.78 & 74.00 & 109.00 & \\
\hline Healthy & 41 & 96.41 & 75.00 & 137.00 & \\
\hline FEV1/FVC(\%)(measured) & & & & & $0.021 *$ \\
\hline Group I & 21 & 80.28 & 69.00 & 88.00 & \\
\hline Healthy & 41 & 84.63 & 72.00 & 99.00 & \\
\hline PEF (L/s) (\%) & & & & & 0.699 \\
\hline Group I & 21 & 88.61 & 54.00 & 115.00 & \\
\hline Healthy & 41 & 88.02 & 65.00 & 121.00 & \\
\hline FEF $25-75(\mathrm{~L} / \mathrm{s})(\%)$ & & & & & 0.303 \\
\hline Group I & 21 & 84.06 & 62.00 & 117.00 & \\
\hline Healthy & 41 & 89.05 & 38.00 & 135.00 & \\
\hline TKA & & & & & $0.003 *$ \\
\hline Group I & 21 & 41.56 & 28.70 & 59.00 & \\
\hline Healthy & 41 & 35.70 & 22.30 & 49.20 & \\
\hline
\end{tabular}

Table 5. Comparison of respiratory function and TKA of the MS patients in Group II and healthy volunteers

\begin{tabular}{|c|c|c|c|c|c|}
\hline & $\mathbf{n}$ & $\%$ & Min & Max & $\mathbf{p}$ \\
\hline FVC (L) (\%) & & & & & $0.001 *$ \\
\hline Group II & 19 & 96.00 & 82.00 & 116.00 & \\
\hline Healthy & 41 & 97.98 & 69.00 & 133.00 & \\
\hline FEV1 (L) (\%) & & & & & $0.000 *$ \\
\hline Group II & 19 & 93.78 & 74.00 & 109.00 & \\
\hline Healthy & 41 & 96.41 & 75.00 & 137.00 & \\
\hline FEV1/FVC (\%)(measured) & & & & & $0.001 *$ \\
\hline Group II & 19 & 80.28 & 69.00 & 88.00 & \\
\hline Healthy & 41 & 84.63 & 72.00 & 99.00 & \\
\hline PEF (L/s) (\%) & & & & & $0.000 *$ \\
\hline Group II & 19 & 88.61 & 54.00 & 115.00 & \\
\hline Healthy & 41 & 88.02 & 65.00 & 121.00 & \\
\hline FEF $25-75(\mathrm{~L} / \mathrm{s})(\%)$ & & & & & $0.000 *$ \\
\hline Group II & 19 & 84.06 & 62.00 & 117.00 & \\
\hline Healthy & 41 & 89.05 & 38.00 & 135.00 & \\
\hline TKA & & & & & $0.000 *$ \\
\hline Group II & 19 & 41.56 & 28.70 & 59.00 & \\
\hline Healthy & 41 & 35.70 & 22.30 & 49.20 & \\
\hline
\end{tabular}

Statistically, a significant difference was detected in the comparison of anthropometric measurements (weight $(\mathrm{kg}), \mathrm{BMI}(\mathrm{kg} / \mathrm{m} 2)$, the circumference of the neck, shoulder, and chest, fathom length) of Group I and Group II MS patients $(p<0.05)$. The average values of these anthropometric measurements of Group I of MS were significantly higher than the average of those of patients in Group II of MS (Table 4).

Pulmonary function test results of the MS patients were compared by dividing them into two groups as patients with TKA values of 400 and below and patients with TKA values of 410 and above. It was found that patients with the TKA value of 400 or down had higher respiratory function test results than patients with the TKA value of 410 or above. There was a statistically significant difference only for FEV1 (L) (\%) and FEF25-75 (L/s) (\%) values (Table 5). Likewise, when the respiratory function test results of the healthy group were compared by dividing them into two groups with a TKA value of 400 and below and those with a TKA value of 410 and above, there was only a statistically significant difference in PEF values $(p=0.018)$.

\section{DISCUSSION}

In this study, clinometric measurements of thoracic curvature and standardized pulmonary function tests are used to assess changes in lung function in MS patients. The female-to-male ratio 
of MS patients who participated in our study was calculated to be $2.3 / 1$, consistent with the incidence rate of female-to-male MS, which is $2.5 / 1$ worldwide (11). In a survey conducted with $238 \mathrm{MS}$ patients comparing body mass index and physical activity and examining the impact of this relationship on neurological disability, no relationship was found between physical activity level and BMI or between obesity and neurological disability (12). In our study, although no statistically significant difference was found in the measurements of BMI, neck and shoulder circumference, and fathom length between MS patients and healthy subjects, the mean chest circumference was statistically significantly higher in MS patients than in healthy subjects. It was hypothesized that the increased thoracic angle caused increased chest cavity size during the MS process.

In MS patients, the motor pathways of the respiratory tract are affected. Therefore respiratory muscle weakness develops, and as a result, ventilatory impairment occurs (13). Initially, the brain can adapt to this respiratory muscle weakness. Thus, respiratory symptoms in neurological diseases usually do not appear until the late stages. The intervening fever or infection further affects the respiratory disorders, the patient's respiratory demand increases, the respiratory muscles become relatively weaker, and the disease's course worsens, as in MS.

Pneumonia is one of the most common pulmonary problems during the disease $(14,15)$. Because pulmonary complications are a significant cause of morbidity and mortality, assessing respiratory muscle and function and supporting them with individualized programs improves both quality of life and patients' lifespan (15).

Howard et al. reported that respiratory complications occurred an average of 5.9 (minmax:1-12) years after the onset of neurologic symptoms. These investigators said severe respiratory problems in 14 patients, and the majority of patients had lesions in the cervical and bulbar regions. Based on these findings, the causes of MS's respiratory disorders were classified into five categories: Respiratory muscle weakness, bulbar dysfunction, obstructive sleep apnea, airway control disorders, and paroxysmal hyperventilation (16). The duration of the disease in MS patients in our study was $8.75 \pm 6.23$ (minmax:1-25) years. This period includes the time to onset of dyspnea in MS patients reported in the literature. For this reason, we think that we evaluate the pulmonary function test results in MS patients with appropriate characteristics.

The decrease in FVC value indicates respiratory dysfunction, which occurs in proportion to the progression of MS. Another study by Smeltzer et al. evaluated the respiratory functions of $25 \mathrm{MS}$ patients with varying degrees of motor dysfunction. It defined two types of respiratory dysfunction: involvement of the respiratory centers in the medulla and acute respiratory failure secondary to cervical spine lesions in MS patients (16).

As MS disease progresses, central respiratory stimulation is increased to maintain partial pressures of carbon dioxide $(\mathrm{CO} 2)$ and oxygen $(\mathrm{O} 2)$ in normal arterial blood. The patient's respiratory rate increases. As the disease progresses, the central adaptive response activates hyperventilation to prevent dyspnea and fatigue. The low vital capacity and decreased airway cleanliness and sigh lead to the development of atelectasis and hypoxemia. This chain of events leads to increased respiratory demand. Although muscle fatigue can lead to tachypnea, alveolar hypoventilation, and acidemia, acute respiratory failure is rarely reported in MS patients $(9,17$, 18). Extensive bulbar involvement may be seen in acute respiratory failure. Due to large plaques in the medulla and cervical spinal segments, the respiratory pattern is disturbed, and apnea and acute respiratory failure are observed. It is often complicated by secondary systemic factors such as dehydration and aspiration pneumonia. Apart from acute complications, the most crucial respiratory dysfunction in patients with MS is an alteration in respiratory function, especially in the expiratory route $(19,20)$. It was found with statistical significance that the values of $\mathrm{PEF}(\mathrm{L} / \mathrm{s})$ (\%) and FEF25-75(L/s) of MS patients in our study were lower than healthy subjects. The low values indicate that MS patients have an expiratory and restrictive outcome. This is in agreement with the findings in the literature. Bosnak et al. reported that expiratory muscles are involved in the early stages of MS. In case of excessive expiratory weakness, cough is inadequate, and secretion clearance is difficult. This leads to respiratory complications, which are the most common cause of morbidity and mortality (21) Many studies have shown that respiratory muscle weakness also develops in 
later stages of MS $(10,20,22)$. There is evidence in the literature that the decrease in exercise tolerance in patients with multiple sclerosis may be related to respiratory muscle dysfunction (10). Providing patients with respiratory and physical activity support at the earliest possible time after diagnosis can lead to positive quality of life outcomes without allowing the respiratory function to deteriorate.

The changes in pulmonary function observed in multiple sclerosis may occur due to respiratory pump dysfunction of the lungs (20). In our study, a statistically significant difference was found between the PEF(L/s)(\%) and FEF25-75(L/s) $(\%)$ values of MS patients and healthy subjects; similar to the results of the study of Altintas et al. These values of healthy subjects were found higher than those of MS patients (21). Our study divided MS patients into two groups: 2.5 and below (group I) and above 2.5 (group II) according to EDDS scores and examined pulmonary functions. The measurements of FVC (L) (\%), FEV1(L) (\%), PEF(L/s) (\%), and FEF25$75(\mathrm{~L} / \mathrm{s})(\%)$ of patients in group I was statistically significantly higher than those of patients in group II. Our study is primarily compatible with the literature regarding the effect of expiratory pattern and restrictive pulmonary involvement (1, 20, 22).

A significant correlation was found between respiratory dysfunction and disease severity (13). Respiratory involvement can be observed in the early stages of the disease, especially during the relapse phases (23). Few studies show that respiratory functioning is impaired in the early stages in MS patients $(18,20)$. Severe cerebellar findings have been found to be associated with respiratory dysfunction in MS patients. Although there are studies showing that respiratory function is impaired in MS patients with high EDSS scores (16), few studies show that respiratory function is impaired in MS patients with low EDSS scores. In the survey conducted by Bosnak et al., impairment of both inspiratory and expiratory muscles was found, and significantly low PEF values were also found in patients with EDSS scores between 2-4.5 (20).

A previous study compared MS patients and healthy volunteers and found abnormalities such as torticollis, the head's anterior tilt, and lordosis. In this study, 30 beneficial and 30 female MS patients with an EDSS score below three were enrolled. Torticollis, scoliosis, lordosis, kyphosis, and an anterior tilt of the head were compared between the two groups. Statistically significant differences in terms of the anterior tilt of the head, torticollis, and lordosis were found, while no statistically significant difference was found between the two groups in thorasic kyphosis (6). Controversially statistically significant difference was found between MS patients and healthy volunteers in terms of kyphosis in our study. Abnormal postures and spastic weakness which can be seen in MS patients may lead to cervical pathologies (24). An increase in the angle of thorasic kyphosis leads to a decrease in the mobility of the thorasic wall and reducing pulmonary capacity. A few studies have been conducted on increased thorasic kyphosis angle and airway restriction $(21,25)$. According to our study results, it was found that pulmonary function tests values decreased with the increased TKA in MS patients. According to our results, the decrease in respiratory function test values with the increase of TKA may indicate a restrictive pattern. Although there are few studies evaluating the effects of thoracal kyphosis angle on airway restriction our result is compatible with the literature (24).

For MS patients, it is essential to maintain their physical activity, functional capacity, independence, and quality of life and to reduce the incidence of comorbidities (26). Traditional exercise programs to increase MS patients' physical activity can be particularly challenging for MS patients with walking difficulties (27). With physical rehabilitation and exercises to be performed under professional guidance and early application of respiratory rehabilitation in MS patients, it can positively influence both respiratory functions and physical activity of patients.

\section{CONCLUSION}

Our results suggest that exceptionally high TKA in MS patients is one of the causes of their impaired lung function. In terms of outcomes, it will be appropriate to involve patients in pulmonary rehabilitation at an early stage of the disease and provide specific treatment programs according to the evaluation of their neck and thoracic region, including respiratory muscles.

Limitations: It would be appropriate to conduct the study with a larger MS patient population and evaluate the patients' osteoporotic characteristics and the thoracic kyphotic angle. 


\section{APPLICABLE REMARKS}

- Since MS's etiological factor is not fully understood, it is a disease that cannot be treated for the etiological factor but can only be managed. In the early phase, respiratory physiotherapy applications should be an essential part of the treatment approach to prevent respiratory pathologies that may occur due to respiratory function impairment and significantly support patients' quality of life.

- Physical activity, especially under professional guidance, provides MS patients with its positive effects on cardiorespiratory and muscular function, significant benefits in maintaining their functional abilities and reducing the incidence of potential comorbidities.

\section{ACKNOWLEDGMENT}

We would like to thank İskender Yilmaz for technical assistance in anthropometric measurements and Doğa Şenan for language control.

\section{CONFLICTS OF INTEREST}

Authors declare no conflict of interest.

\section{FINANCIAL DISCLOSURES}

On behalf of all co-authors, I confirm that I have reviewed and complied with the relevant Instructions to Authors, the Ethics in Publishing policy, and Conflicts of Interest disclosure. I also assign to the journal the copyright for my manuscript and any other data intended for publication in the manuscript. I will not submit or publish any part in any form or language if the article is accepted for publication. I have informed the co-author(s) of this publishing agreement's terms and that I am signing on their behalf as their agent, and I am authorized to do so.

\section{ETHICAL CONSIDERATIONS}

The study's ethics committee approval was obtained from the Scientific Research and Publication Ethics Board of Dr. Burhan Nalbantoğlu State Hospital (dated 20.06.2018 and decision number 2018 / 25-12). Also all participants have signed a consent form to participate in the study.

\section{REFERENCES}

1. Levy J, Prigent H, Bensmail D. Respiratory rehabilitation in multiple sclerosis: A narrative review of rehabilitation techniques. Ann Phys Rehabil Med. 2018;61(1):38-45. doi: 10.1016/j.rehab.2017.06.002 pmid: 29128525

2. Kalron A. Gait variability across the disability spectrum in people with multiple sclerosis. J Neurol Sci. 2016;361:1-6. doi: 10.1016/j.jns.2015.12.012 pmid: 26810506

3. Broekmans T, Gijbels D, Eijnde BO, Alders G, Lamers I, Roelants M, et al. The relationship between upper leg muscle strength and walking capacity in persons with multiple sclerosis. Mult Scler. 2013;19(1):112-119. doi: 10.1177/1352458512444497 pmid: 22562952

4. Soysal Tomruk M, Uz MZ, Kara B, Idiman E. Effects of Pilates exercises on sensory interaction, postural control and fatigue in patients with multiple sclerosis. Mult Scler Relat Disord. 2016;7:70-73. doi: 10.1016/j.msard.2016.03.008 pmid: 27237762

5. Guner S, Haghari S, Inanici F, Alsancak S, Aytekin G. Knee muscle strength in multiple sclerosis: relationship with gait characteristics. J Phys Ther Sci. 2015;27(3):809-813. doi: 10.1589/jpts.27.809 pmid: 25931736

6. Ebrahimi Meymand F, Daneshmandi H, Sahib Al-Zamani M, Ebrahimi HA. Spinal Cord Analysis in People with Multiple Sclerosis. Ann Appl Sport Sci. 2018;6(1):29-35. doi: 10.29252/aassjournal.6.1.29

7. Koele MC, Lems WF, Willems HC. The Clinical Relevance of Hyperkyphosis: A Narrative Review. Front Endocrinol (Lausanne). 2020;11:5. doi: 10.3389/fendo.2020.00005 pmid: 32038498

8. Sangtarash F. Validity and Reliability of Dual Digital Inclinometer in Measuring Thoracic Kyphosis in Women over 45 Years. J Spine. 2013;03(03). doi: 10.4172/2165-7939.1000170

9. Fry DK, Pfalzer LA, Chokshi AR, Wagner MT, Jackson ES. Randomized control trial of effects of a 10week inspiratory muscle training program on measures of pulmonary function in persons with multiple sclerosis. J Neurol Phys Ther. 2007;31(4):162-172. doi: 10.1097/NPT.0b013e31815ce136 pmid: 18172412

10. Foglio K, Clini E, Facchetti D, Vitacca M, Marangoni S, Bonomelli M, et al. Respiratory muscle function and exercise capacity in multiple sclerosis. Eur Respir J. 1994;7(1):23-28. doi: 10.1183/09031936.94.07010023 pmid: 8143827 
11.Eskandarieh S, Nedjat S, Abdollahpour I, Moghadasi AN, Azimi AR, Sahraian MA. Comparing epidemiology and baseline characteristic of multiple sclerosis and neuromyelitis optica: A case-control study. Mult Scler Relat Disord. 2017;12:39-43. doi: 10.1016/j.msard.2017.01.004 pmid: 28283104

12. So WY, Kalron A. The Association between Body Mass Index and Leisure-Time Physical Activity in Adults with Multiple Sclerosis. Int $J$ Environ Res Public Health. 2020;17(3). doi: 10.3390/ijerph17030920 pmid: 32024246

13. Grasso MG, Lubich S, Guidi L, Rinnenburger D, Paolucci S. Cerebellar deficit and respiratory impairment: a strong association in multiple sclerosis? Acta Neurol Scand. 2000;101(2):98-103. doi: 10.1034/j.1600-0404.2000.101002098.x pmid: 10685856

14. Sadovnick AD, Eisen K, Ebers GC, Paty DW. Cause of death in patients attending multiple sclerosis clinics. Neurology. 1991;41(8):1193-1196. doi: 10.1212/wnl.41.8.1193 pmid: 1866003

15. Vincken W, Elleker MG, Cosio MG. Determinants of respiratory muscle weakness in stable chronic neuromuscular disorders. Am J Med. 1987;82(1):53-58. doi: 10.1016/0002-9343(87)90377-9 pmid: 3799694

16. Howard RS, Wiles CM, Hirsch NP, Loh L, Spencer GT, Newsom-Davis J. Respiratory involvement in multiple sclerosis. Brain. 1992;115 ( Pt 2):479-494. doi: 10.1093/brain/115.2.479 pmid: 1606478

17. Martin-Valero R, Zamora-Pascual N, Armenta-Peinado JA. Training of respiratory muscles in patients with multiple sclerosis: a systematic review. Respir Care. 2014;59(11):1764-1772. doi: 10.4187/respcare.02881 pmid: 24825913

18. Bosnak-Guclu M, Gunduz AG, Nazliel B, Irkec C. Comparison of functional exercise capacity, pulmonary function and respiratory muscle strength in patients with multiple sclerosis with different disability levels and healthy controls. $J$ Rehabil Med. 2012;44(1):80-86. doi: 10.2340/16501977-0900 pmid: 22234321

19. Smeltzer SC, Utell MJ, Rudick RA, Herndon RM. Pulmonary function and dysfunction in multiple sclerosis. Arch Neurol. 1988;45(11):1245-1249. doi: 10.1001/archneur.1988.00520350083020 pmid: 3190505

20. Buyse B, Demedts M, Meekers J, Vandegaer L, Rochette F, Kerkhofs L. Respiratory dysfunction in multiple sclerosis: a prospective analysis of 60 patients. Eur Respir J. 1997;10(1):139-145. doi: 10.1183/09031936.97.10010139 pmid: 9032506

21. Klefbeck B, Hamrah Nedjad J. Effect of inspiratory muscle training in patients with multiple sclerosis. Arch Phys Med Rehabil. 2003;84(7):994-999. doi: 10.1016/s0003-9993(03)00133-3 pmid: 12881823

22. Mullen AE, Wilmarth MA, Lowe S. Cervical disk pathology in patients with multiple sclerosis: two case reports. Phys Ther. 2012;92(8):1055-1064. doi: 10.2522/ptj.20110004 pmid: 22517783

23. Tantucci C, Massucci M, Piperno R, Betti L, Grassi V, Sorbini CA. Control of breathing and respiratory muscle strength in patients with multiple sclerosis. Chest. 1994;105(4):1163-1170. doi: 10.1378/chest.105.4.1163 pmid: 8162744

24.Lorbergs AL, O'Connor GT, Zhou Y, Travison TG, Kiel DP, Cupples LA, et al. Severity of Kyphosis and Decline in Lung Function: The Framingham Study. J Gerontol A Biol Sci Med Sci. 2017;72(5):689694. doi: 10.1093/gerona/glw124 pmid: 27341855

25.Lombardi I, Jr., Oliveira LM, Mayer AF, Jardim JR, Natour J. Evaluation of pulmonary function and quality of life in women with osteoporosis. Osteoporos Int. 2005;16(10):1247-1253. doi: 10.1007/s00198-005-1834-3 pmid: 15806323

26. Coulter EH, Bond S, Dalgas U, Paul L. The effectiveness of interventions targeting physical activity and/or sedentary behaviour in people with Multiple Sclerosis: a systematic review. Disabil Rehabil. 2020;42(5):594-612. doi: 10.1080/09638288.2018.1503737 pmid: 30290702

27. Aminian S, Motl RW, Rowley J, Manns PJ. Management of multiple sclerosis symptoms through reductions in sedentary behaviour: protocol for a feasibility study. BMJ Open. 2019;9(4):e026622. doi: 10.1136/bmjopen-2018-026622 pmid: 30940762 